\title{
„La citation pour la citation“
}

D ie Evidence Based Medicine hat wesentlich zu einem Qualitätsgewinn beigetragen. $\mathrm{Da}$ bei gilt es aber immer wieder die Grenzen zu beachten und Auswüchse zu vermeiden. Eine unglückliche Entwicklung ist das ständige und unkritische Zitieren; dies geht so weit, dass wir alle, die nicht jede Aussage mit einem Zitat belegen, des Plagiats beschuldigen. Allgemeines Lehrbuchwissen muss nicht unbedingt mit einem Zitat belegt werden und die Tatsache, dass man eine Aussage mit einer Quelle belegen kann, heißt noch lange nicht, dass die Aussage korrekt ist oder richtig verstanden wurde. Umgekehrt werden manche Aussagen als definitiv angesehen, obwohl sie nie geäußert wurden. Ein berühmtes Beispiel ist das Zitat „Beam me up, Scotty", das alle kennen, aber im entsprechenden Film nie gesagt wurde.

\section{Aus einer anderen Welt}

$\mathrm{Zu}$ Beginn meiner beruflichen Laufbahn lebten wir noch in einer anderen Welt. Wir haben lange nach Quellen gesucht und waren froh, wenn wir den vermeintlichen Erstbeschreiber entdeckt hatten und die historische Arbeit in Händen hielten. Diese Quelle haben wir natürlich gerne und voller Stolz zitiert. Ein besonderer Erfolg war es zeigen zu können, dass eine Neuerung schon lange vorher in einer anderen Sprache publiziert war (idealerweise der eigenen). Allgemeine Aussagen mussten nicht mit Zitaten belegt werden, da auch die Quelle diese Aussage meist ohne sinnvollen Beleg getroffen hatte, und es war sogar möglich, eine persönliche Mitteilung zu zitieren. Heute sind wir hingegen der Ansicht, dass man weder allgemeine Aussagen treffen noch eigene Ideen haben darf. Immer wieder sehe ich, dass als Quelle allgemeiner Aussagen Lehrbücher oder Reviews zitiert werden. Wenn ich meine langjährige Erfahrung in einen Lehrbuchbeitrag einfließen lassen möchte, muss ich erst noch ein $\mathrm{Zi}$ tat suchen, um das zu belegen, was bei der großen Flut von Publikationen allerdings kein Problem mehr darstellt.
Die vielen „open access journals“ machen es möglich, dass eine Arbeit bei wiederholtem Einreichen irgendwann abgedruckt wird und damit zitiert werden kann. Gab es früher das Problem, dass gute Arbeiten unter Wert oder eventuell gar nicht veröffentlicht wurden, besteht heute die Gefahr, dass wertlose und nicht überprüfbare Daten publiziert werden. Durch das Internet sind diese Arbeiten schnell und überall verfügbar, jedoch wird selten die Richtigkeit der Ergebnisse angezweifelt. Ist ein gutes Printjournal nicht überall verfügbar, wird es immer seltener zitiert, da die Aussage auch leichter mit einem anderen Zitat (eventuell Sekundär- oder Tertiärliteratur) belegt werden kann.

\section{"Und man kann ausgemachte Lügner zitieren, solange man sie nur korrekt zitiert."}

Siri Hustvedt, "The Blazing world“ (2014)

Haben wir früher Arbeiten zum Thema gelesen, gesammelt und dann in die Diskussion einfließen lassen, könnten wir heute eine Diskussion frei formulieren und die Aussagen durch entsprechende Quellen belegen. Ist die komplette Arbeit nicht verfügbar, wird eventuell nur die Zusammenfassung gelesen. Selbst wenn die Ergebnisse von Studien längst relativiert oder gar widerrufen wurden, passiert es, dass Arbeiten weiter zitiert werden.

Wir könnten uns aber auf das Wesentliche beschränken. Die Anzahl der Referenzen korreliert ja nicht unbedingt mit der Qualität. Eigentlich bräuchte man nur konkrete Zahlen und/oder die daraus folgenden Aussagen zu zitieren. Idealerweise nehmen wir hier Bezug auf die Originalquellen und 


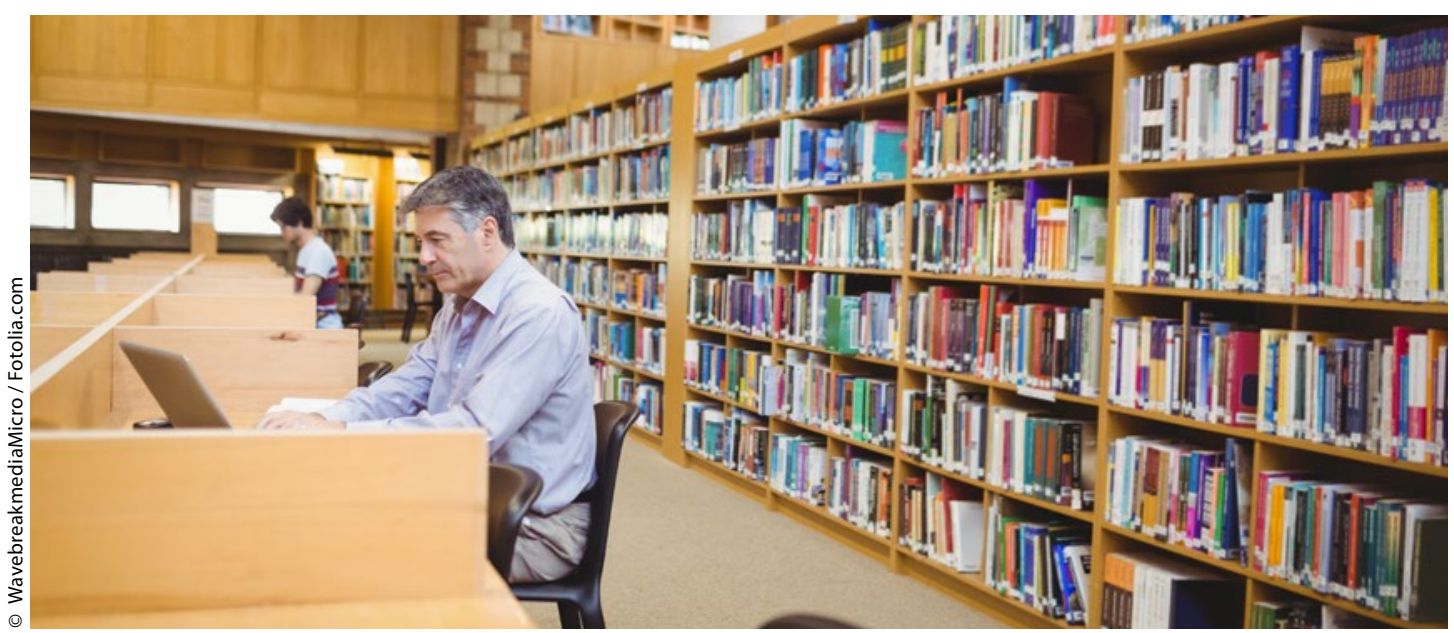

Ist ein gutes Printjournal nicht überall verfügbar, wird es immer seltener zitiert, da die Aussage auch leichter mit einem anderen Zitat belegt werden kann.

nicht auf Übersichtsarbeiten oder die erste Publikation in englischer Sprache. Die Zeitschriften sollten darauf achten, dass Originalzitate auch richtig wiedergegeben werden. Oft passiert es, dass sich bei der redaktionellen Überarbeitung grobe Fehler bei Zitaten anderer Sprache einschleichen und diese dadurch nicht von Suchmaschinen erfasst werden.

\section{Von Interessenskonflikten und Ghostwritern}

In diesem Zusammenhang kann man auch die Angabe der Interessenskonflikte kritisch sehen. Die aktuelle Vorgehensweise schützt vor gar nichts und schafft auch nicht mehr Offenheit. Wenn ein Autor Interessenskonflikte angibt, ist die Welt in Ordnung, der Form wurde Genüge getan und eine weitere Überprüfung findet nicht statt.

Die Aufzählung der Firmen, bei der der Autor Berater ist oder Vorträge gegen Honorar hält, offenbart doch nicht, ob die Arbeit dadurch beeinflusst wurde und in welcher Form. Diese Listen am Ende der Publikationen werden immer länger und unlesbarer. Nicht selten werden Stellen von der Industrie finanziert, die fast nie angegeben werden, selbst wenn der Autor persönlich davon betroffen ist. Auch hier wäre Offenheit, Klarheit und Ehrlichkeit gefragt. Wir wissen alle, dass viele Arbeiten immer noch von Ghostwritern geschrieben werden und das nicht offengelegt wird. In Acknowledgement und in den Interessenskonflikten sind Allgemeinplätze wenig hilfreich, konkrete Angaben wären sinnvoll. Eine nette Vorlage zeigt uns Gary Perlman im Journal of Irreproducible Results 1991; 27: 29-30: „I owe nothing to anyone concerning this research. I did it all myself. Maureen Graham of the St. Andrew's University did not inspire me at all, not a bit. Anyway, she has left the country."
Weder meine Meinung noch dieser Text werden an der Vorgehensweise etwas ändern. Ich befürchte sogar, dass sich die Zustände noch verschlimmern, da die richtige Zitierweise und der einfache Verweis und Interessenskonflikte leicht überprüft werden können, die Richtigkeit einer Aussage aber nur mit großem Aufwand. Wir haben schon lange Probleme, qualifizierte Gutachter für die Journale zu finden, wie sollen diese auch noch die Quellen kontrollieren. Aber wir müssen daran arbeiten, sonst verlieren wir unsere Glaubwürdigkeit. Wenn die Aussage einer Arbeit unkritisch übernommen und damit weitere Ansätze und Hypothesen sowie Aussagen begründet werden, kann kein sinnvolles Ergebnis folgen. Der falsche Weg führt nur selten zum Ziel.

„Denn was man schwarz auf weiß besitzt, kann man getrost nach Hause tragen."

J. W. Goethe, Faust, Teil 1

Herzliche kollegiale Grüße

Ihr

Wolfgang Jost

Prof. Dr. med. Wolfgang Jost

Parkinson-Klinik Ortenau

Kreuzbergstr. 12-24, 77709 Wolfach

E-Mail:w.jost@parkinson-klinik.de 